\title{
Abordaje multidisciplinar al tumor de saco endolinfático
}

L.G. González-Bonet*,**; L.I Amoros-Sebastiá***; J. Piquer-Belloch*; P. Riesgo*; J.L. Llacer*; J. GisbertAguilar*** y L. Morell-Quadreny****

Servicios de Neurocirugía*, Otorrinolaringología*** y Anatomía Patológica****. Hospital de La Ribera. Alzira. Valencia. Servicio de Neurocirugía** Hospital General de Castellón. Castellón.

Resumen

Introducción. El adenocarcinoma papilar de saco endolinfático se asocia a la enfermedad de Von Hippel Lindau en el $\mathbf{1 5 \%}$ de los casos, tiene un crecimiento lento pero elevada agresividad local, y no metastatiza. Clínicamente produce un Síndrome de Menière derivado de la compresión que provoca en el conducto endolinfático. Cuando debuta con pérdida de audición suele ser de forma brusca e irreversible. Se diagnostica con técnicas de imagen y el análisis de su estructura con inmunohistoquímica. Su tratamiento electivo es la exéresis quirúrgica, y su principal complicación el sangrado perioperatorio, que se puede evitar con embolización o radiación estereotáctica preoperatorio.

Caso clínico. Presentamos un caso de un tumor de saco endolinfático en un paciente de 17 años aquejado de sordera unilateral y crisis de vértigo rotatorio, con antecedentes familiares de enfermedad de Von Hippel Lindau. Las pruebas complementarias mostraron una hipoacusia neurosensorial y una arreflexia vestibular derechas. En tomografía computarizada de peñascos se apreciaba una lesión en el acueducto vestibular. Se sometió al paciente a una petrosectomía con abordaje presigmoideo y preservación de laberinto, realizándose una exéresis total de la lesión. Se diagnosticó de tumor del saco endolinfático en el análisis anatomopatológico.

Discusión. Ante un paciente con antecedentes familiares de enfermedad de Von Hippel Lindau y un cuadro clínico de vértigo incluso sin hipoacusia, o siendo esta leve, habría que pensar en la presencia de un tumor del saco endolinfático. La presentación clínica de sordera puede ser brusca e irreversible, incluso con imágenes negativas o poco concluyentes, por lo que una rápida actuación es importante para la preservación de esta función.

PALABRAS CLAVE. Enfermedad de Von Hippel Lindau. Hipoacusia neurosensorial. Petrosectomía. Tumor de peñasco. Tumor del saco endolinfático.

Recibido: 6-05-11. Aceptado: 4-08-11
Multidisciplinary approach to the endolymphatic sac tumour

Summary

Introduction. Papilar adenocarcinoma of endolymphatic sac is related with Von Hippel Lindau disease at $15 \%$ of cases, has a slow growing with a high local aggressiveness, and doesn't metastasize. It causes symptoms of Meniere's syndrome due to the compression that produces at endolymphatic duct. When it presents with hearing loss is usually sudden and irreversible manner. The diagnostic is made with image tests and analysis of its structure with immunohistochemical tests. The elective treatment is surgical remove, and its main complication the perioperative bleeding it can be avoided with preoperative embolization or stereotactic radiation.

Case report. A case of endolymphatic sac tumour is presented, in a 17-years-old male with unilateral deafness and crisis of rotate vertigo, with family history of Von Hippel-Lindau disease. Perceptive deafness and right vestibular arreflexia are detected at technical exploration. In a petrous bone computer tomography appears a mass at vestibular aqueduct. We performed a petrosectomy with presigmoidal approach and saving of inner ear. Pathological analysis revealed an endolymphatic sac tumour.

Discussion. In patients with a family history of Von Hippel Lindau disease and clinical symptoms of vertigo and normal hearing or with slight hearing loss we should suspect the presence of endolymphatic sac tumor. The clinical presentation of hearing loss can be sudden and irreversible even with negative or inconclusive images. Therefore, a quick action is important for the preservation of this function.

Abreviaturas. EVHL: enfermedad de Von Hippel Lindau. TSE: tumor de saco endolinfático. RNM: Resonancia nuclear magnética. TC: tomografía computarizada. 
KEY WORDS. Endolymphatic sac tumour. Neurosensorial hearing loss. Petrosectomy. Petrous bone tumour. Von Hippel-Lindau disease.

\section{Introducción}

Los tumores del saco endolinfático son tumores localmente invasivos del hueso temporal que aunque pueden presentarse de forma aislada se asocian en un $15 \%$ de frecuencia a la Enfermedad de Von Hippel Lindau (EVHL) ${ }^{14}$. Se caracterizan clínicamente por presentar pérdida de audición siendo esta en muchas ocasiones de forma irreversible y súbita. También pueden producir vestibulopatía e incluso alteraciones del nervio facial. La alteración de la audición se puede producir incluso antes de que las pruebas de imagen detecten la lesión tumoral. Esta pérdida de audición súbita se ha relacionado con la producción de una hemorragia o un hidrops endolinfático ${ }^{10}$.

Todos estos datos obligan a que este tipo de lesiones poco frecuentes tengan que estar presentes en el "screening" de un paciente con EVHL incluso sin clínica de pérdida de audición pero, mucho más, en los que ya han comenzado con ella.

En este trabajo, presentamos un caso de un tumor del saco endolinfático, abordado de forma multidisciplinar por ORL y Neurocirugía, donde una rápida actuación permitió la mejora de la clínica del paciente que tan pocas veces ocurre $^{19}$. Además, repasamos la historia natural y el origen anatómico hasta hace poco desconocido de este tipo de lesiones, revisamos los mecanismos que subyacen a la temprana clínica, e intentamos optimizar el "timing" del diagnóstico y tratamiento para reducir la incidencia y gravedad de la disfunción neurológica que provocan estos tumores.

\section{Caso clínico}

Varón de 17 años que presenta, como antecedentes personales, reacción adversa a metoclopramida con sintomatología extrapiramidal, rinitis alérgica y cirugía ortopédica de rodilla. Como antecedentes familiares se asocia padre fallecido a causa de EVHL. Acude a consulta por hipoacusia leve subjetiva de oído derecho y 4 crisis de vértigo rotatorio de unos 3 días de duración en el último año, sin antecedentes previos de mareo.

En la exploración clínica se aprecia otoscopia normal bilateral. La audiometría muestra una hipoacusia neurosensorial en oído derecho de $65 \mathrm{~dB}$ en graves y 20 $\mathrm{dB}$ en agudos, con normoacusia en oído izquierdo. El timpanograma es normal. En la exploración vestibular constatamos un sistema sacádico normal, sin disimetrías ni adiadococinesia, con ausencia de nistagmus espontáneo y con mínimo nistagmus horizontal a la derecha en posición de la mirada hacia abajo. Pruebas de Romberg y Barany sin

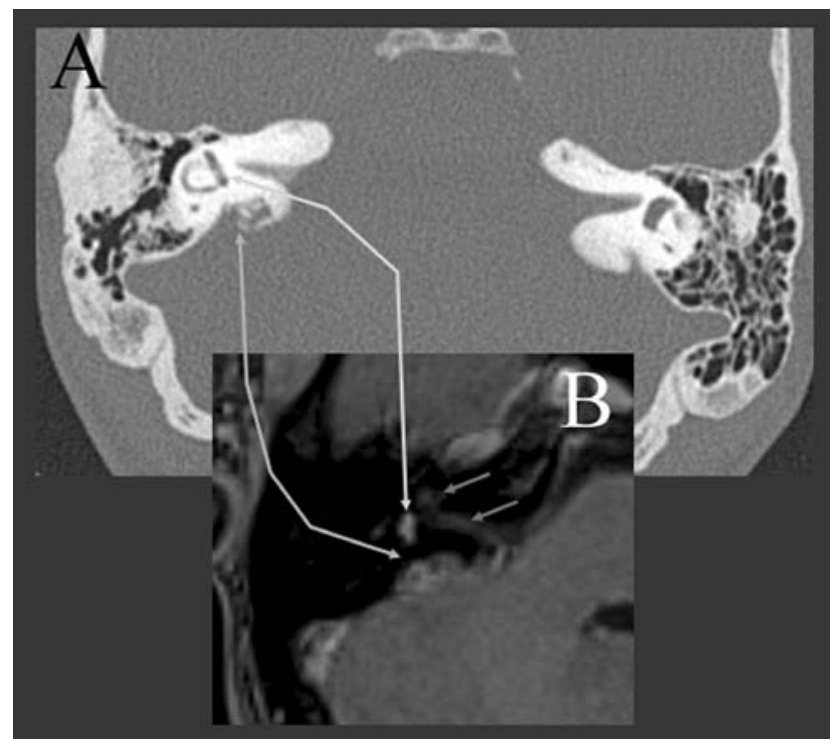

Figura 1. A: tomografía computarizada donde se observa una masa en el espesor de porción petrosa retrolaberíntica del hueso temporal derecho. B: imagen de resonancia magnética en $T 1$ con contraste donde se observa una lesión captante de forma heterogénea. Así mismo, se muestra la correlación de la imagen con la tomografia.

alteraciones. Marcha en estrella y Fukuda con lateropulsión y spin a la derecha mínimo. En la videonistagmografía, con la exploración del laberinto posterior, las pruebas calóricas ponen de manifiesto una arreflexia vestibular derecha compensada $(2,3 \%$ def $57 \%)$. En las pruebas posicionales no hay nistagmus de posición.

La exploración oftalmológica muestra una agudeza visual correcta (1.0/1.0), con polo anterior y fondo de ojo normales y sin lesiones.

Se solicita una TC de peñascos, apreciándose una lesión en la porción petrosa del hueso temporal derecho, centrada en el acueducto vestibular (figura 1A). En la RNM secuenciada en T1 con gadolinio se observa una captación de contraste heterogénea en la zona anteriormente nombrada de la TC (figura 1B). El diagnóstico diferencial se plantea entre tumor del saco endolinfático y glomus yugular. Por frecuencia se trataría de un glomus yugular pero la localización centrada en el acueducto vestibular y la edad del paciente sugieren más un tumor del saco endolinfático. El resto del oído medio es normal.

Con el diagnóstico de presunción de tumor de saco endolinfático se practica un abordaje presigmoideo respetando el bloque laberíntico. Se aprecia tumoración extradural muy vascularizada, que no parece infiltrar dura, aspirándose la misma, practicándose apertura dural con resección, y advirtiéndose hipertrofia dural sin infiltración tumoral. Se practica hemostasia cuidadosa y sellado del defecto dural con plastia de dura e injerto graso más cola 


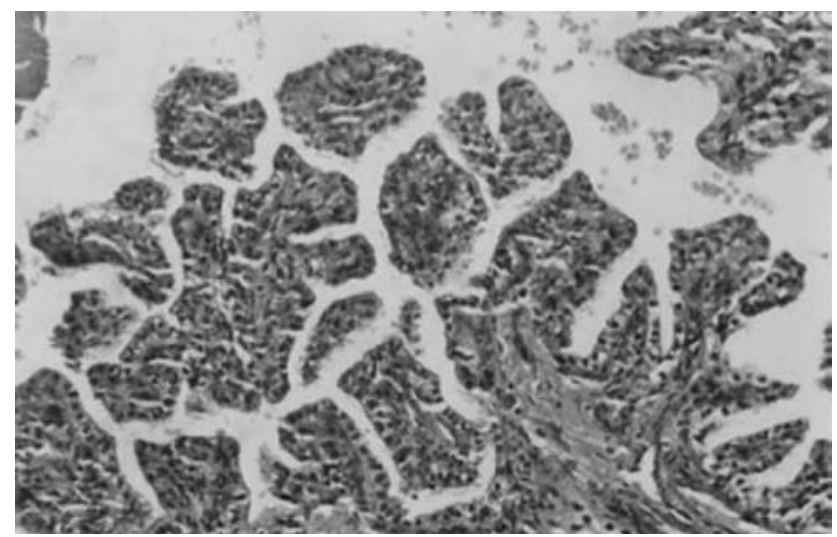

Figura 2. Tinción de hematoxilina-eosina donde se observan estructuras papilares con contenido eosinófilo amorfo y un estroma conectivo denso. Ambas estructuras se hallan tapizadas por un epitelio cúbico con escaso citoplasma.

de fibrina y cierre por planos.

En la histología de la pieza se halla sobre el estroma conectivo denso, pequeñas proyecciones papilares $\mathrm{y}$ estructuras foliculares llenas de material amorfo eosinófilo, ambas revestidas por epitelio cúbico, con escaso citoplasma claro y núcleos homogéneos sin atipias ni mitosis evidentes (figura 2). El índice proliferativo es bajo, con marcaje de Ki 67 en aproximadamente el 1\% de las células tumorales, que presentan intensa positividad frente a citoqueratina y vimentina, y moderada inmunotinción frente a EMA; habiendo resultado negativas frente a cromogranina, desmina, CD34 y tiroglobulina. Se diagnóstica por ello de tumor de saco endolinfático.

Ambulatoriamente el paciente fue sometido a estudio genético confirmándose la EVHL.

El postoperatorio transcurrió sin complicaciones y con audición medida por audiometría similar a la preoperatoria. Al mes el audiograma tonal muestra una recuperación completa de los niveles auditivos. En los controles sucesivos del paciente a 3, 6 y 12 meses, no se observa recidiva de la lesión.

\section{Discusión}

El saco y conducto endolinfático son estructuras del laberinto membranoso llenas de líquido endolinfático y derivadas embriológicamente del neuroectodermo. El conducto endolinfático es un corto canal que pasa por detrás del vestíbulo a través del acueducto vestibular óseo hasta desembocar en el saco endolinfático. El saco endolinfático está compuesto de una compleja red de túbulos interdigitados con criptas y papilas. Esta organización es más compleja en la parte media o rugosa del saco, que es donde se cree que se originan los tumores del saco ${ }^{11}$. En nuestro caso, los hallazgos encontrados durante la cirugía, en la histología y en las pruebas de imagen son concluyentes con ese origen.

Hyams y Michaels describieron por vez primera los adenomas de oído medio en 1976. El adenoma de saco endolinfático fue descrito en 1988 por Gaffey, si bien fue Hassard el primero en sugerir en 1984 que tales tumores podían proceder de esa localización ${ }^{7}$. Su estructura ha generado en ocasiones confusión con otro tipo de tumores, como papilomas del plexo coroideo extradural, tumores de glándula ceruminal ectópicas y adenocarcinoma metastásico de origen renal o tiroideo ${ }^{11}$. Durante muchos años existió una importante controversia acerca de su origen histológico, hasta que Heffner, en 1989, estableció que se trataba de un adenocarcinoma papilar procedente del saco endolinfático, razón por la que también se le conoce como tumor de Heffner $^{8}$. Heffner estudió la ultraestructura e inmunohistioquímica de 20 tumores papilares en comparación con el saco endolinfático normal y propuso éste como localización del tumor. Posteriores estudios parecen confirmar esta hipótesis ${ }^{4}$, si bien la controversia persiste ${ }^{21}$. Algunos autores sostienen que los tumores del saco endolinfático podrían tratarse de una variante del encapsulado adenoma del oído medio, o proceder de tejido neuroectodérmico en oído medio de las células de la cresta neural o bien de depósitos ectópicos ${ }^{15}$.

Aunque puede presentarse espontáneamente, el tumor del saco endolinfático se asocia a la EVHL en el 11-15\% de los $\operatorname{casos}^{6,11,13,17,23}$. De hecho, los casos bilaterales de tumor del saco endolinfático únicamente han aparecido asociados a esta patología, suponiendo aproximadamente el $30 \%$ de los casos de EVHL con tumor de saco endolinfático ${ }^{16}$.

La EVHL es una alteración genética rara, caracterizada por la predisposición al desarrollo de tumores malignos o benignos viscerales y del sistema nervioso central: angiomas de retina, hemangioblastomas del sistema nervioso central y cerebelo, troncoencéfalo, espina y nervios (incluyendo el tumor de saco endolinfático), feocromocitomas, carcinomas de células renales, cistoadenomas de anexos reproductivos, tumores de células del islote pancreático o neuroendocrinos y quistes benignos afectando otros órganos ${ }^{13,15,23}$. Cualquier paciente, como el presentado por nosotros, con tumor del saco endolinfático es en principio subsidiario de un estudio genético, de igual modo que los pacientes con EVHL deben someterse a resonancias magnéticas de conducto auditivo interno periódicas para diagnosticar tempranamente la aparición de un tumor de saco endolinfático ${ }^{11}$. Los casos de síndrome de Menière de evolución tórpida y mala respuesta al tratamiento pueden esconder también un tumor de saco endolinfático, haciéndose mandatario en estos casos una exploración radiológica exhaustiva de peñascos ${ }^{15}$. Tiene una prevalencia de aproximadamente 1:40.00023. Es más frecuente en mujeres, habiéndose descrito casos entre los 4 y 71 años $^{11}$. La asociación de EVHL con el tumor del 
saco endolinfático frente a la aparición espontánea de éste se asocia a menor edad (30 años de media frente a 52 años), mayor incidencia en mujeres sobre varones $(2: 1$ frente a $1: 1)$, bilateralidad (27:1) y mayor grado de diferenciación $(90 \% \text { grados I-II frente a } 83 \%)^{16}$. La EVHL se transmite por herencia autonómica dominante con penetración incompleta ${ }^{13,23}$. La mutación afecta a un gen supresor de tumores localizado en el cromosoma 3 p25 y es causante de la constelación de tumores del cuadro ${ }^{23}$. A su vez, el estudio genético del tumor de saco endolinfático ha arrojado diversas alteraciones, como la sustitución de guanina (G) por timina (T) en el nucleótido 564 que resulta en una sustitución del aminoácido Trp a Cys, delección de un par de bases en el exómero 1 del gen del Von Hippel-Lindau y pérdida de heterocigosidad en el cromosoma 3 p25.5, locus del gen de Von Hippel-Lindau ${ }^{12}$.

La presencia de una masa destructiva en peñasco plantea un reto diagnóstico. Normalmente el diagnóstico diferencial se presenta con otras masas destructoras de peñasco principalmente paragangliomas ${ }^{11}$. Histológicamente el tumor del saco endolinfático se trata de un adenocarcinoma de bajo grado que se origina en la porción proximal o rugosa del saco endolinfático ${ }^{5,16}$. También se describen a veces como tumores papilares con áreas organizadas de fibrosis y hemorragias o bien quísticas ${ }^{11}$. Se trata de tumores vasculares localmente agresivos que pueden invadir estructuras vecinas en el hueso temporal, pero que no metastatizan ${ }^{23}$. Presenta dos tipos diferenciados: el folicular, con aspecto reminiscente de parénquima tiroideo y preponderancia de quistes coloides ${ }^{8,15}$ que suele estar delimitado, y el papilar, más sólido y agresivo, que puede dar lugar a destrucción local y penetración en fosa posterior ${ }^{11}$. Este último caso es el visto por nosotros en nuestro caso clínico. De todas formas, ambos tipos pueden hallarse de forma mixta en el mismo tumor ${ }^{8}$. El tumor puede presentar material coloide que se cree está relacionado con el fluido proteináceo de la porción rugosa del saco endolinfático.

Usualmente se trata de tumores de crecimiento lento y localmente invasivos ${ }^{11}$. Las lesiones avanzadas se extienden más frecuentemente en la fosa craneal posterior $^{22}$, pero también anteriormente en el seno cavernoso, superiormente en la fosa craneal media e inferiormente en el foramen yugular ${ }^{11,22}$, pudiendo llegar a destruir completamente el hueso temporal u occipital ${ }^{1}$. Clásicamente no se conocen metástasis de este tumor ${ }^{11}$, si bien en 2002 Ferreira informó de un tumor de saco endolinfático que debutó como metástasis ${ }^{5}$. Su lento crecimiento provoca que presenten historias de evolución local a largo plazo (30 años o más $)^{1,22}$.

Clínicamente produce síntomas indistinguibles de la enfermedad de Menière (triada de Menière: hipoacusia, vértigo y acúfenos) debido a la obstrucción del conducto endolinfático que produce ${ }^{2,23}$, siendo predominante la sor- dera neurosensorial unilateral. Esta pérdida de audición es brusca en el $43 \%$ de los casos, progresiva en un periodo de 3 a 6 meses en otro $43 \%$ y progresiva más lentamente en el resto ${ }^{14}$. Típicamente la pérdida de audición es irreversible y ocurre muy pronto (en una media de edad de 22 años). Esto normalmente coincide con el pico de síntomas vestibulares.

Otros síntomas que puede producir son: parálisis del nervio facial ${ }^{11,13}$, otalgia ${ }^{17}$, disfunción del nervio facial $o$ plenitud auricular ${ }^{13}$. Incluso se ha descrito como masa en conducto auditivo externo o en ángulo pontocerebeloso, causando hidrocefalia ${ }^{8}$. Se podría plantear en ese caso el estudio de imagen de peñasco de los pacientes con Menière resistentes a tratamiento. En un estudio se demostró que alrededor del $0,3 \%$ de los síndromes de Menière estaban causados por tumoración del saco endolinfático ${ }^{2}$.

Normalmente los síntomas cocleovestibulares se producen por afectación tumoral del oído interno y orientan sobre la evolución del crecimiento tumoral. Tumores microscópicos indetectables radiológicamente ${ }^{14}$, que no afectan la cápsula ótica pueden, no obstante, producir síntomas vestibulares o sordera. Esto se cree que es debido a fenómenos de hemorragia tumoral o hydrops ${ }^{13}$. Por otra parte la lentitud de crecimiento de los tumores permitiría teóricamente la compensación de las lesiones directas por destrucción ósea sobre laberinto, de forma que la sintomatología clínica no siempre se corresponde en intensidad correlativamente con el tamaño del tumor ${ }^{15}$. Esto obliga, como presentamos en nuestro caso, a una actuación rápida y multidisciplinar para un diagnóstico y tratamiento precoz. De esta forma ante la sospecha razonable de tumor de saco endolinfático por pequeños indicios como eran, la clínica imprecisa, la erosión del peñasco en la TAC y los antecedentes familiares del paciente, procedimos a una valoración quirúrgica que consiguió la preservación de la audición del paciente.

Por su parte, la anatomía patológica conlleva incluso el análisis genómico para detectar mutaciones ${ }^{23}$. La inmunohistoquímica puede mostrar positividad hacia anticuerpos enolasa específicos, factor de crecimiento endotelial vascular, antineurona, vimentina, S100, anti antígeno de membrana epitelial o proteína ácida fibrilar de la glía ${ }^{8,9,11,15}$. Podemos hallar positividad para citoqueratinas CAM 5.2, 34betaE-12, CK7, CK8 y CK 19 pero no para CK 10/13 o CK20. En áreas focales se puede hallar inmunorreactividad para el antígeno Ki-67 (MIB-1) ${ }^{9}$. Se diagnostica un tumor del saco endolinfático por reacción positiva a marcadores de neuroectodermo y epiteliales y negativa a marcadores neuronales ${ }^{11}$.

Radiológicamente el tumor se presenta básicamente como una lesión destructora de hueso ${ }^{15,19,23}$. En la resonancia magnética se puede apreciar un realce de la masa en T1 y un realce heterogéneo en T2. Las lesiones gran- 
des pueden mostrar imágenes líquidas correspondientes a $\operatorname{vasos}^{8,23}$. La hiperintensidad en T1 es bastante específica del tumor de saco endolinfático frente a otras lesiones que asientan en peñasco. Normalmente la masa se presenta heterogénea y predominantemente hiperintensa, con posibles calcificaciones o imágenes de vasos, incluso fragmentos de hueso ${ }^{11}$. Las zonas hiperintensas corresponden a hemorragias subagudas, áreas quísticas proteináceas o acúmulos de colesterol. Es frecuente observar un anillo de hiperintensidad en el borde de la lesión ${ }^{11}$. La administración de contraste intravenoso provoca generalmente el realce heterogéneo de las lesiones, tanto en RNM como en $\mathrm{TAC}^{11}$. Se localiza típicamente, en las fases tempranas, entre el seno sigmoide y el conducto auditivo interno, esto es, en la localización del saco endolinfático ${ }^{11}$.

Las pruebas radiológicas revisten suma importancia pues hay que diferenciar el diagnóstico de tumor de saco endolinfático no solo con el de paraganglioma sino también con el de otras lesiones que asientan en el hueso temporal, como metástasis de carcinoma renal, hepático, pulmón o tiroides; meningiomas; papilomas de plexo coroideo; lesiones óseas primarias y secundarias o tumores adenomatosos del oído medio ${ }^{15}$.

La angiografía mostrará una vascularización variable, procedentes bien de ramas de la arteria carótida externa (faríngea ascendente, auricular posterior y rama posterior de la meníngea media) o bien de la rama meníngea posterior de la arteria vertebra ${ }^{11}$. En los tumores más grandes puede aparecer nutrición de la carótida interna o de la circulación posterior, dependiendo de la dirección de crecimiento del tumor ${ }^{11}$.

El tratamiento quirúrgico, pese a la dificultad de extirpación completa (debido a su naturaleza agresiva local), se mantiene como el principal método de tratamiento ${ }^{8}$. Las indicaciones para el tratamiento quirúrgico se basan principalmente en la capacidad de curar completamente el tumor preservando la función cocleovestibular en lo posible. En aquellos pacientes con síntomas pero sin evidencia radiológica de tumor, se indicará la intervención quirúrgica, en el caso de hallazgos radiológicos sugestivos de hemorragia focal ${ }^{13}$. Los tumores pequeños asintomáticos del saco endolinfático se deben intervenir para impedir que en el futuro generen vértigo, acúfeno o sordera. Los pacientes que sufran una cofosis tendrán indicación quirúrgica si existe compromiso neurológico ${ }^{13}$. El tratamiento quirúrgico se puede efectuar por medio de un abordaje retrosigmoideo, si bien se suele practicar una exploración transmastoidea ${ }^{11,15,16}$. La petrosectomía presigmoidea o retrolaberíntica posterior permite una excelente visión de las estructuras anatómicas específicas $^{13,18}$, como el hueso petroso posterior, el ducto y el saco endolinfático. Ambos abordajes, no obstante, pueden usarse combinados. Es recomendable la monitorización intraoperatoria del nervio facial y también de los potenciales evocados del tronco cerebral si existen restos auditivos detectados preoperatoriamente ${ }^{13}$. La técnica comienza con una mastoidectomía cortical clásica, procediendo, una vez delimitado el tegmen, el seno sigmoide y el laberinto óseo, a una exposición de la duramadre de la fosa posterior, identificando el saco endolinfático por debajo de la línea de Donaldson y el ducto endolinfático se encuentra dentro del acueducto vestibular óseo ${ }^{13}$. Normalmente los tumores pequeños se hallan en la porción más distal del conducto endolinfático ${ }^{13}$. Se procede a la escisión tumoral, así como de las celdas petrosas adyacentes hasta que se halla hueso sano. Igualmente se extirpa la duramadre de fosa posterior hasta que se alcanzan unos márgenes de seguridad suficientes. La operación se concluye obliterando el antro con fascia o tejido adiposo, y el defecto dural con grasa y pegamento de fibrina ${ }^{13}$. El sangrado intraoperatorio es la complicación que más frecuentemente puede hacer fracasar la intervención ${ }^{6}$.

En ocasiones pueden ser precisas varias intervenciones. Algunos autores recomiendan la radiación estereotáctica preoperatoria para evitar el sangrado que en ocasiones hace fracasar la cirugía. Anatomopatológicamente se ha demostrado que la radioterapia preoperatoria facilita la fibrosis del tejido intersticial y los vasos del tumor ${ }^{6}$.

La embolización preoperatoria es una opción que se debe valorar a la luz del diámetro de los vasos que nutren al tumor y con el objeto de reducir la pérdida hemática intraoperatoria. Se puede utilizar partículas de alcohol polivinilo ${ }^{1}$. El seguimiento se puede realizar tanto con TAC de alta resolución como con $\mathrm{RNM}^{13}$. Cohen presentó en 2003 un protocolo de tratamiento con angioplastia asistida por stent de la carótida petrosa, embolización selectiva endovascular y extirpación quirúrgica, con el objeto de reducir la posibilidad de sangrado ${ }^{3}$.

Un estudio de Kim con 5 pacientes intervenidos por vía retrolaberíntica posterior refiere mejoría de los síntomas de vértigo, acúfeno y plenitud aural, con preservación de la audición preoperatoria ${ }^{13}$. La probabilidad de preservación de la audición se incrementa si el tumor es pequeño o in situ, factor importante en el caso de los pacientes afectos de EVHL con más probabilidades de presentar tumor bilateral ${ }^{16}$.

La serie de casos conocidos de tumores del saco endolinfático es demasiado corta para sacar conclusiones, pero parece evidente que la recidiva tras resección completa es más improbable que tras resección parcial del tumor, con o sin radioterapia postoperatoria. Sin embargo, el diagnóstico se suele realizar en estadios avanzados, haciendo difícil en muchas ocasiones una exéresis completa. En caso de ser sólo posible la resección parcial, y dado el lento ritmo de crecimiento del tumor, se hace obligatorio un seguimiento postoperatorio superior a una década ${ }^{11}$. En caso de recidiva 
algunos autores utilizan la radiocirugía con gamma-knife, si bien no libra al paciente del tumor ${ }^{5}$.

El uso de la radioterapia es controvertido, debido a que estos tumores raramente presentan malignidad en el estudio anatomopatológico y no se conocen casos de metástasis $^{20}$.

\section{Conclusión}

En los pacientes afectos de EVHL, evitar la aparición de sordera y desequilibrio que generan, entre otras, sus diversas manifestaciones tumorales es el reto clínico más importante. El diagnóstico precoz y un adecuado tratamiento quirúrgico a tiempo pueden ser claves en el manejo de estos pacientes.

El diagnóstico apropiado es aquel que se basa en los fenómenos clínicos, las pruebas radiológicas, los hallazgos operatorios, así como los resultados histológicos e inmunohistoquímicos.

En aquellos pacientes con sospecha clínica y radiológica de tumores del saco endolinfático, se puede evitar la instauración de una sordera permanente y conservar la audición mediante una resección quirúrgica usando una petrosectomía posterior retrolaberíntica presigmoidea.

\section{Bibiliografía}

1. Asano, K., Sekiya, T., Hatayama, T., et al.: A case of endolymphatic sac tumor with long-term survival. Brain Tumor Pathol. 1999; 16: 69-76.

2. Cmejrek, R.C., Megerian, C.A.: Obstructing lesions of the endolymphatic sac and duct mimicking Meniere's disease. Ear Nose Throat J. 2004; 83: 753-756.

3. Cohen, J.E., Spektor, S., Valarezzo, J., Fellig, Y., Umansky, F.: Endolymphatic sac tumor: staged endovascularneurosurgical approach. Neurol Res. 2003; 25: 237-240.

4. Devaney, K.O., Ferlito, A., Rinaldo, A.: Endolymphatic sac tumor (low-grade papillary adenocarcinoma) of the temporal bone. Acta Otolaryngol. 2003; 123: 1022-1026

5. Ferreira, M.A., Feiz-Erfan, I., Zabramski, J.M., Spetzler, R.F., Coons, S.W., Preul, M.C.: Endolymphatic sac tumor: unique features of two cases and review of literature. Acta Neurochir (Wien). 2002; 144: 1047-1053.

6. Hashimoto, M., Yolota, A., Urasaki, E., Imada, H., Yamamoto, H.: Surgical treatment of endolymphatic sac tumor with adjunctive stereotactic radiation therapy-case report. Neurol Med Chir (Tokyo). 2004; 44: 595-599.

7. Hassard, A.D., Boudreau, S.F., Cron, C.C.: Adenoma of the endolymphatic sac. J Otolaryngol. 1984; 13: 213-216.

8. Heffner, D.K.: Low-grade adenocarcinoma of probable endolymphatic sac origin A clinicopathologic study of 20 cases. Cancer. 1989; 64: 2292-2302

9. Horiguchi, H., Sano, T., Toi, H., Kageji, T., Hirokawa, M.,
Nagahiro, S.: Endolymphatic sac tumor associated with a Von Hippel-Lindau disease patient: an mmunohistochemical study. Mod Pathol. 2001; 14: 727-732.

10 Jeffrey, K.H., Butman, J.A., Bewer, C., Zalewski, C., Vortmeyer, A.O.: Tumours of the endolymphatic sac in patients with Von Hippel-Lindau disease: implications for their natural history, diagnosis and treatment. J Neurosurg. 2005; 102: 503-512.

11. Joy, H.M., Barker, C.S., Millar, J.S., Davis, A.: Radiological considerations in the diagnosis of an endolymphatic sac tumour. Clin Radiol. 2002; 57: 652-660.

12. Kawahara, N., Kume, H., Ueki, K., Mishima, K., Sasaki, T., Kirino, T.: VHL gene inactivation in an endolymphatic sac tumor associated with von Hippel-Lindau disease. Neurology. 1999; 53: 208-210.

13. Kim, H.J., Butman, J.A., Brewer, C. et al.: Tumors of the endolymphatic sac in patients with von Hippel-Lindau disease: implications for their natural history, diagnosis, and treatment. J Neurosurg. 2005; 102: 503-512.

14. Lonser, R.R., Kim, H.J., Butman, J.A., Vortmeyer, A.O., Choo, D.I., Oldfield, E.H.: Tumors of the endolymphatic sac in Von Hippel-Lindau disease. NEJM. 2004; 305: 2481-2486.

15. Luff, D.A., Simmons, M., Malik, T., Ramsden, R.T., Reid, H.: Pathology in focus. Endolymphatic sac tumours. J Laryngol Otol. 2002; 116: 398-401.

16. Megerian, C.A., Haynes, D.S., Poe, D.S., Choo, D.I., Keriakas, T.J., Glasscock, M.E. 3rd. Hearing preservation surgery for small endolymphatic sac tumors in patients with von Hippel- Lindau syndrome. Otol Neurotol. 2002; 23: 378-387.

17. Minteguiaga, C., García Ibañez, L., Tran Ba Huy, P..: Tumor del saco endolinfático y enfermedad de Von Hippel Lindau. Revisión de la literatura. Acta Otorrinolaringol Esp. 2002; 53: 515-520.

18. Oghalai, J.S., Jackler, R.K.: Anatomy of the combined retrolabyrinthine-middle fossa craniotomy. Neurosurg Focus. 2003 Jun 15; 14: e8.

19. Peyre, M., Gaillard, S., van Effenterre, R., Giraud, S., Richard, S.: Conservative management of endolymphatic sac tumors in von Hippel-Lindau disease: case report.. Acta Neurochir (Wien). 2011 Jan; 153: 42-47; discussion 47. Epub 2010 Nov 20.

20. Polinsky, M.N., Brunberg, J.A., McKeever, P.E., Sandler, H.M., Telian, S., Ross, D.: Aggressive papillary middle ear tumours: a report of two cases with review of the literature. Neurosurgery.1994; 35: 493-497.

21. Pollack, A., Bohmer, A., Spycher, M., Fisch, U.: Are papillary adenomas endolymphatic sac tumours? Ann Otol Rhinol Laryngol. 1995; 104: 613-619.

22. Reijneveld, J., Hanlo, P., Groenewoud, G., Jansen, G., van Overbeeke, K., Tulleken, C.: Endolymphatic sac tumor: a case report and review of the literature. Surg Neurol. 1997; 48: 368-373.

23. Williamson, R.A., Coker, N.J.: Endolymphatic sac tumor in Von Hippel-Lindau disease. Otol and Neurol. 2003; 28: 832 . 
González-Bonet, L.G.; Amoros-Sebastiá, L.I.; PiquerBelloch, J.; Riesgo, P.; Llacer, J.L.: Gisbert-Aguilar, J.; Morell-Quadreny, L.: Abordaje multidisciplinar al tumor de saco endolinfático. Neurocirugía 2011. 22: 567-573.
Correspondencia: Luis Germán González Bonet. Servicio de Neurocirugía. Hospital General de Castellón. Avda de Benicassim s/n 12004

Email: gonzalez_luibon@gva.es 